\title{
Foetal Kidney Length Can Be A New Parameter For Determination of Gestational Age in Third Trimester \\ N.I.Ashmawy ${ }^{1}$, T.M.Assar ${ }^{1}$, A.S.Saad ${ }^{1}$ and A.M.Omar ${ }^{2}$ \\ ${ }^{1}$ Obstetrics, Gynecology Dept., Faculty of Medicine, Benha Univ., Benha, Egypt \\ ${ }^{2}$ Obstetrics, Gynecology Dept., Faculty of Medicine, Beheria Univ., Beheria, Egypt \\ E-Mail:amany3omar.ao@gmail.com
}

\begin{abstract}
Gestational age assurance is one of the best strides in the administration of the timing of pregnancy and subsequent diminishing in perinatal mortality due to preterm conveyances. There is no single boundary to assess the gestational age. The utilization of ultrasound (US) biometric routine boundaries, including biparietal width (BPD), head outline (HC), stomach circuit, femoral length (FL), and fetal weight, is obligatory at all pregnancies beginning from 20 weeks. However, at late incubation, these boundaries are not exact. This examination expected to assess the precision of fetal kidney length by ultrasonography in assessing gestational age in third trimester. Techniques: This investigation is cross sectional examination that was done at Benha University clinic during the period from March 2019 to March 2020.Routine U/S assessment and kidney assessment. Estimations of all fetal biometric boundaries were acquired. Results: When contrasting the determined GA and LMP with various boundaries, the mean of LMP GA was (36.5 \pm 2.6 ), BPD GA was (36.0 \pm 2.72$), \mathrm{HC}$ GA was $(35.8 \pm 2.70)$, AC GA was $(35.5 \pm 2.9)$, FL GA was (36.5 \pm 2.73$)$. There were measurably critical positive connection between's mean fetal kidney length and (biparietal breadth (BPD), BPD GA, head outline (HC), HC GA, stomach boundary (AC), AC GA, femoral length (FL) and FL GA. The current investigation determined a condition for assessment of the gestational age from the fetal kidney length (FKL) with SE \pm 2.4 days. End Fetal kidney length increments with increment in fetal gestational age (FGA) and shows incredible intra and bury class connection coefficient which recommends great understanding and reproducibility of estimations.
\end{abstract}

Keywords: Foetal kidney length, Ultrasonography, Gestational age-third trimester.

\section{Introduction}

Gestational age affirmation is perhaps the best step in the organization of the circumstance of pregnancy and ensuing reducing in perinatal mortality due to preterm transports. There is no single boundary to assess the gestational age. Essentially, likewise with all disclosures and advancements, it is continually fundamental to look for better and more accommodating frameworks with an open and enquiring mind. In studying the gestational age, the fetal kidney length opens up the probability of finding the gestational age with a singular boundary [1].

Exact assurance of gestational age assumes a noteworthy job of decrease of postdate works, which improves both fetal and maternal consideration and diminishes postnatal confusion. It is the best way to know the satisfactory planning of compulsory intercessions and the evasion of right on time or late mediations [2].

The utilization of ultrasound (US) biometric routine boundaries, including biparietal measurement (BPD), head circuit (HC), stomach perimeter, femoral length $(\mathrm{FL})$, and fetal weight, is required at all pregnancies beginning from 20 weeks [3]; notwithstanding, late at pregnancy, every one of these boundaries are not extremely exact attributable to either troublesome specialized issues, intrauterine development hindrance, or muddled pregnancy. These estimations indicated critical inconsistency, which was seen with every day practice particularly late at third trimester [4]

Numerous investigations have been acted in the previous twenty years concerning the sonographic appraisal of fetal kidney length (FKL). At early examination stages, numerous investigations were accomplished for conclusion of fetal renal contortion and later on, they were done to evaluate the relationship among's FKL and gestational age [5].

Numerous creators suggested utilizing a FKL as another boundary since it isn't influenced by the error of late trimester or by fetal development hindrance [6].

FKL estimation is more exact at late pregnancy due to a specialized issue. At early pregnancy, the kidneys and suprarenal organs have the comparable appearance of echogenicity and possibly inspirable, however at the third trimester, the perinephric fat turns out to be more evident, which makes the diagram of the kidneys effectively detachable from contiguous structures particularly the suprarenal organ [7].

FKL has a consistent development of $1.7 \mathrm{~mm}$ fortnightly through pregnancy [4].

This examination meant to assess the exactness of fetal kidney length by ultrasonography in assessing gestational age in third trimester.

\section{Patients and methods}

Type of the study: Cross sectional study.

Study settings: Benha University hospital.

Ethical consideration: the study was affirmed by Ethical Committee of the division of Obstetrics and Gynecology personnel of medication Benha University. educated assent was gotten from every member.

Study populace: 100 cases were selected among cases going to Obstetrics and Gynecology division of benha college clinic of for Ante natal consideration during the period from March 2019 till March 2020. 
Consideration models: Healthy ladies who were sure of their LMP and had earlier ordinary menstrual cycles with simple pregnancy between 28 weeks of incubation to 40 weeks were chosen for the investigation. Dating of pregnancy since early pregnancy (first trimester)was pointedly known dictated by both estimating of crown back end length (CRL) and sure LMP which agree with gestational age.

Avoidance standards: Oligohydramnios. Polyhydramnios. Diabetic mother. Pregnancy actuated hypertension. Various developments. Fetal variations from the norm. Renal irregularities. IUGR. Undefined adrenal or renal fringes.

\subsection{Methodology}

\section{A definite history}

Routine risk natal examinations was finished.

Examination( general, Obstetrics, conceivable neighborhood)

Routine U/S assessment and kidney assessment.

Estimations of all fetal biometric boundaries was acquired utilizing a continuous, grayscale, 3.5-5.0 $\mathrm{MHz}$ curvilinear cluster transducer. GE voluson 730 ace $v$.

An agreeable cross over plane of the hatchling was first characterized at the degree of the four offices of the heart, following which cross over filtering was proceeded in a cephalocaudal course until the fetal kidneys were imagined, regularly at the degree of the stomach, or just beneath it. The test was then arranged through $90^{\circ}$ to get the longitudinal pivot of every kidney on either side of the midline cylindrical anechoic stomach aorta. The biggest longitudinal picture indicating both prevalent and sub-par external shafts of every kidney was gotten and solidified on the screen. Utilizing electronic calipers, kidney length was estimated from the better external shaft than the substandard external post [8].

Three estimations was taken per kidney to limit intraobserver mistake, and the normal incentive in millimeters was recorded in a worksheet. Care was taken to prohibit the adrenal organs (AGs) from the estimations . Gestational age(GA) in weeks was resolved from the patient's LMP utilizing Naegele's rule,thus speaking to GA by LMP, while the assessed GA was resolved from the Hadlock's outline of anticipated fetal estimations at explicit menstrual weeks for BPD, HC, FL, and AC, utilizing all around characterized reference focuses. The determined Gestational Age (CGA) in weeks, was inferred as a normal of the deliberate biometric lists (BPD, HC, FL, and $\mathrm{AC}$ ), were comparably report in the worksheet. Every estimation was completed multiple times and the normal was figured and recorded as the mean worth. [9].

Gestational age was determined from mean Fetal kidney length (FKL) [10].This esteem was then contrasted and genuine GA got from sure LMP determined mean GA done by fetal biometry.

\subsection{Statistical analysis}

The gathered information were organized and investigated utilizing SPSS rendition 24 delicate product (SpssInc, Chicago, ILL Company. Unmitigated information were introduced as number and rates while quantitative information were communicated as mean \pm standard deviation, middle and range. Quantitative information were tried for typicality utilizing ShapiroWilks test, expecting ordinariness at $\mathrm{P}>0.05$. Friedman test was utilized to coordinate GA dependent on LMP with the determined and anticipated GA. Friedman test was trailed by post hoc various examinations utilizing Bonferroni balanced Wilcoxon test to identify the coordinated sets. Non parametric relationships were surveyed by Spearman's connection coefficient (rho). Straightforward direct relapse investigation was hurried to define a condition for rediction of GA from mean fetal kidney length. The acknowledged degree of noteworthiness in this work was expressed at 0.05 ( $\mathrm{P}$ $\leq 0.05$ was thought of significant).

\section{Results}

Table (1) Age and anthropometric measurements of the studied sample.

\begin{tabular}{lcc}
\hline Variable $(\mathbf{n}=\mathbf{1 0 0})$ & Mean \pm SD & Range \\
\hline Age $($ years) & $24.4 \pm 5.1$ & $16-44$ \\
Weight $(\mathbf{k g})$ & $70.3 \pm 9.9$ & $53-105$ \\
Height $(\mathbf{c m})$ & $165.7 \pm 4.9$ & $150-175$ \\
BMI $\left(\mathbf{k g} / \mathbf{m}^{2}\right)$ & $25.5 \pm 2.6$ & $20.2-34.3$ \\
\hline
\end{tabular}

The mean of age $(24.4 \pm 5.1)$ years, weight $(70.3 \pm 9.9) \mathrm{kg}$, height $(165.7 \pm 4.9) \mathrm{cm}$ and BMI $(25.5 \pm 2.6)(\mathrm{kg} / \mathrm{m} 2) \mathrm{Table}(1)$.

Table (2) Fetal biometric parameters and Calculated gestational ages among the studied sample.

\begin{tabular}{lccc}
\hline Variable $(\mathbf{n = 1 0 0})$ & Mean \pm SD & Median & Range \\
\hline BPD $(\mathbf{m m})$ & $87.7 \pm 8.9$ & 89.9 & $48.3-98.6$ \\
HC $(\mathbf{m m})$ & $322.8 \pm 18.1$ & 327.6 & $269.3-345.6$ \\
AC $(\mathbf{m m})$ & $316 \pm 29.4$ & 323.2 & $239-360.8$ \\
FL $(\mathbf{m m})$ & $71.1 \pm 8.9$ & 72.5 & $36.1-96.8$ \\
Length of rt kidney $(\mathbf{m m})$ & $38.06 \pm 3.27$ & 37.8 & $30.6-48.7$ \\
\hline
\end{tabular}




\begin{tabular}{llcc}
\hline Table (2) Continue & & & \\
\hline Length of lt kidney (mm) & $37.5 \pm 3.42$ & 37.9 & $30-50.5$ \\
Mean length of both kidneys (mm) & $37.7 \pm 3.09$ & 37.5 & $30.7-49.7$ \\
\hline
\end{tabular}

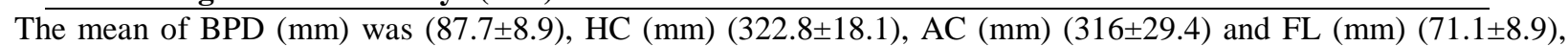
Length of rt kidney was (38.06 \pm 3.27$)$, Length of lt kidney was (37.5 \pm 3.42$)$, Mean length of both kidneys (mm) was (37.7 \pm 3.09$)$ Table ( 2 ).

Table ( 3) Calculated gestational ages among the studied group.

\begin{tabular}{lccc}
\hline Variable (n=100) & Mean \pm SD & Median & Range \\
\hline BPD GA (w) & $36.0 \pm 2.72$ & 36.4 & $28 \mathrm{w}, 4 \mathrm{~d}-40 \mathrm{w}, 3 \mathrm{~d}$ \\
HC GA (w) & $35.8 \pm 2.70$ & 36.3 & $28 \mathrm{w}-39 \mathrm{w}, 4 \mathrm{~d}$ \\
AC GA (w) & $35.5 \pm 2.9$ & 36.2 & $28 \mathrm{w}-39 \mathrm{w}, 6 \mathrm{~d}$ \\
FL GA (w) & $36.5 \pm 2.73$ & 37.1 & $29 \mathrm{w}-40 \mathrm{w}, 3 \mathrm{~d}$ \\
\hline
\end{tabular}

The mean of BPD GA (w) was (36.0 \pm 2.72$)$, HC GA (w) was (35.8 \pm 2.70$)$, AC GA (w) was (35.5 \pm 2.9$)$ and FL GA (w) was (36.5 \pm 2.73$)$ Table ( 3 ).

Table ( 4 ) Comparing the calculated Ga with LMP GA.

\begin{tabular}{lcccc}
\hline Variable (n=100) & Mean $\mathbf{t S D}$ & Median & Range & $\begin{array}{c}\text { Multiple comparisons by } \\
\text { Bonferroni adjusted } \\
\text { Wicoxon (p value) }\end{array}$ \\
\hline LMP GA (w) & $36.5 \pm 2.6$ & 37.0 & $29 \mathrm{w}-40 \mathrm{w}, 1 \mathrm{~d}$ & Reference \\
BPD GA (w) & $36.0 \pm 2.72$ & 36.4 & $28 \mathrm{w}, 4 \mathrm{~d}-40 \mathrm{w}, 3 \mathrm{~d}$ & $4.5(<0.001)$ \\
HC GA (w) & $35.8 \pm 2.70$ & 36.3 & $28 \mathrm{w}-39 \mathrm{w}, 4 \mathrm{~d}$ & $6.27(<0.001)$ \\
AC GA (w) & $35.5 \pm 2.9$ & 36.2 & $28 \mathrm{w}-39 \mathrm{w}, 6 \mathrm{~d}$ & $6.91(<0.001)$ \\
FL GA (w) & $36.5 \pm 2.73$ & 37.1 & $29 \mathrm{w}-40 \mathrm{w}, 3 \mathrm{~d}$ & $0.72(0.47)$ \\
Friedman test (p value) & & $134.1(<0.001, \mathrm{HS})$ & \\
\hline
\end{tabular}

The mean of LMP GA was (36.5 \pm 2.6$)$, BPD GA was (36.0 \pm 2.72$)$, HC GA was (35.8 \pm 2.70$)$, AC GA was (35.5 \pm 2.9$)$, FL GA was (36.5 \pm 2.73$)$ Table ( 4 ).

Table ( 5 ) Correlation between mean fetal kidney length and the studied variables.

\begin{tabular}{lcc}
\hline With & \multicolumn{2}{c}{ Mean fetal kidney length (mm) } \\
\cline { 2 - 3 } & rho & $\mathrm{P}$ \\
\hline Age (years) & -0.12 & 0.23 \\
Weight (kg) & 0.079 & 0.43 \\
Height (cm) & 0.234 & $0.019(\mathrm{~S})$ \\
BMI (kg/m $\mathbf{2}^{\mathbf{2}}$ & -0.028 & 0.78 \\
Gravidity & -0.214 & $0.033(\mathrm{~S})$ \\
Parity & -0.170 & 0.17 \\
No of abortions & -0.09 & 0.68 \\
GA LMP (w) & 0.397 & $<0.001(\mathrm{HS})$ \\
BPD (mm) & 0.220 & $0.028(\mathrm{~S})$ \\
BPD GA (w) & 0.315 & $0.001(\mathrm{HS})$ \\
HC (mm) & 0.354 & $<0.001(\mathrm{HS})$ \\
HC GA (W) & 0.330 & $0.001(\mathrm{HS})$ \\
AC (mm) & 0.296 & $0.003(\mathrm{~S})$ \\
AC GA (W) & 0.338 & $0.001(\mathrm{HS})$ \\
FL (mm) & 0.429 & $<0.001(\mathrm{HS})$ \\
FL GA (W) & 0.363 & $<0.001(\mathrm{HS})$ \\
\hline
\end{tabular}

There were statistically significant positive correlation between mean fetal kidney length and (Height, Gravidity, GA LMP, BPD, BPD GA, HC, HC GA, AC, AC GA, FL and FL GA, and there was no statistically significant difference between mean fetal kidney length and other numerical data Table (5). 
Table (6) Simple linear regression analysis and equation to predict Gestational age from mean fetal kidney length.

\begin{tabular}{|c|c|c|c|c|c|c|}
\hline \multirow[t]{2}{*}{ Model summary } & \multirow{2}{*}{\multicolumn{2}{|c|}{$\begin{array}{c}\mathbf{R}^{2} \\
\mathbf{0 . 1 9 5}\end{array}$}} & Adjusted R ${ }^{2}$ & \multirow{2}{*}{$\frac{\text { SEE }}{2.4}$} & \multirow{2}{*}{$\begin{array}{c}\mathbf{F} \\
23.7\end{array}$} & \multirow{2}{*}{$\begin{array}{c}\text { P-value } \\
\text { 0.001(HS) }\end{array}$} \\
\hline & & & 0.187 & & & \\
\hline \multirow[t]{2}{*}{ Variable } & \multicolumn{2}{|c|}{$\begin{array}{l}\text { Unstandardized } \\
\text { Coefficients }\end{array}$} & $\begin{array}{c}\text { Standardized } \\
\text { Coefficients }\end{array}$ & $95 \%$ CI of $B$ & $\mathbf{t}$ & $\mathbf{P}$ \\
\hline & B & Std. Error & Beta & & & \\
\hline$\left(\right.$ Constant, $\left.b_{0}\right)$ & 22.1 & 2.95 & --- & $16.2-27.9$ & 7.48 & $<0.001(\mathrm{HS})$ \\
\hline $\begin{array}{l}\text { Mean length of } \\
\text { both } \\
\text { kidneys }\end{array}$ & 0.38 & 0.078 & 0.442 & $0.23-0.53$ & 4.87 & $<0.001(\mathrm{HS})$ \\
\hline Equation: & \multicolumn{6}{|c|}{$\begin{array}{c}\mathrm{Y}=\mathrm{b}_{0}+\mathrm{Xb} \\
\mathrm{GA}=22.1+0.38 \times \text { Mean kidney length }(\mathrm{mm})\end{array}$} \\
\hline
\end{tabular}

Derived an equation for estimation of the gestational age from the FKL with SE \pm 2.4 days Table (6).

\section{Discussion}

This investigation indicated that, the mean of length of right kidney was (38.06 \pm 3.27$)$, Length of left kidney was $(37.5 \pm 3.42)$, Mean length of the two kidneys $(\mathrm{mm})$ was $(37.7 \pm 3.09)$. Kansaria, [11] found no huge contrast between the privilege and left kidney lengths. These examiners completed their estimations. Their exploration was a longitudinal report wherein sequential estimations were done at 2 week by week spans in every member (in an Asian populace).

Be that as it may, Edevbie and Akhigbe, [9] in their examination Ultrasound estimation of fetal kidney length in typical pregnancy and relationship with gestational age. The mean left FKL was marginally yet altogether higher than the mean right FKL over the different long stretches of incubation $(\mathrm{P}<0.05)$. The mean consolidated FKL (MKL) was $32.18 \pm 5.96 \mathrm{~mm}$.

This examination demonstrated that, when contrasting the determined GA and LMP with various boundaries, the mean of LMP GA was $(36.5 \pm 2.6)$, BPD GA was (36.0 \pm 2.72$)$, HC GA was $(35.8 \pm 2.70)$, AC GA was $(35.5 \pm 2.9)$, FL GA was $(36.5 \pm 2.73)$. This outcomes showing that, the absolute most exact boundary for assessing GA is the fetal kidney length.This concurs with Uger et al., [12] who discovered mean fetal biometry boundaries including BPD of the investigation populace was $31.97 \pm 4.35$ weeks, FL was $31.76 \pm 4.36$ weeks, $\mathrm{HC}$ was $31.98 \pm 4.32$ weeks, and AC was $31.62 \pm 4.75$ weeks. The mean FKL estimation of patients was $35.66 \pm 6.61 \mathrm{~mm}$ (go $19-49 \mathrm{~mm}$ ). Edevbie and Akhigbe [9] found that, FKL can fill in as an extra solid boundary for This examination demonstrated that, there were factually critical positive connection between's mean fetal kidney length and gestational age by date (relationship coefficient $\mathrm{r}=$ 0.39; $\mathrm{P}<0.001)$. This concurs with Abdelrazek et al., [5] who found that, there is a solid noteworthy connection between's the normal kidney length and gestational age by date (relationship coefficient $\mathrm{r}=$ 0.83 ; $\mathrm{P}<0.05)$.

This investigation indicated that, there were factually noteworthy positive relationship between's mean fetal kidney length and (biparietal width (BPD), BPD GA, head outline (HC), HC GA, stomach periphery (AC), AC GA, femoral length (FL) and FL GA. The emphatically sure noteworthy connection between's MKL in millimeters and GA in weeks as anticipated by BPD, HC, FL, and $\mathrm{AC}$ in this investigation underpins the discoveries of a few different analysts. [13]

In the current examination, there were factually critical positive connection between's GA by LMP (w) and biparietal measurement (BPD), head circuit (HC), stomach boundary (AC) and femoral length (FL). This concurs with Uger et al., [12] who announced the gestational age (GA) was assessed by early fetal ultrasound measures and last menstrual period. Routine fetal biometric boundaries, fetal kidney length, and amniotic liquid record were estimated. There was a solid positive connection among's GA and BPD $(\mathrm{r}=0.975, \quad \mathrm{p}=0.001), \quad \mathrm{HC} \quad(\mathrm{r}=0.974, \quad \mathrm{p}=0.001), \quad \mathrm{FL}$ $(\mathrm{r}=0.967, \mathrm{p}=0.001)$, and $\mathrm{AC}(\mathrm{r}=0.852, \mathrm{p}=0.001)$.

The current investigation determined a condition for assessment of the gestational age from the FKL with SE \pm 2.4 days. The approval consequences of relapse condition have been contrasted and study finished with Konje et al., [14] with SE 10.29 days; Kansaria \&Paruleker, [11].

This examination demonstrated that, relapse model for FKL to anticipate GA is (R2=0.44). This concurs with Uger et al., [12] who found that, relapse model for FKL to foresee GA is (R2=0.896), while relapse model for AFI alone to foresee GA has a more unfortunate presentation $(\mathrm{R} 2=0.249)$. At last, fetal kidney length is a doable estimation with high prescient worth that can be utilized as an aide boundary. Fetal kidney length can foresee a fastidious date of GA in babies who are vulnerable to development issues or when the normal boundaries are absent, for example, achondroplasia, phocomelia, amelia, fetal hepatosplenomegaly, cranial agenesis, or anencephaly.

\section{Conclusion}

Fetal kidney length increments with increment in FGA and shows incredible intra and entombs class relationship coefficient which proposes great understanding and reproducibility of estimations. 


\section{References}

[1] M.SHIRAZ AKRAM, U.FAROOQI, N.ARIF. Estimation of Gestational Age from Fetal Kidney Length in the Second and Third Trimester of Pregnancy by Ultrasonography. Saudi Pharmaceutical J., Vol.5, PP. 222229,2019.

[2] K.BUTT, K.LIM. Diagnostic Imaging Committee. Determination of gestational age by ultrasound. J Obstet Gynaecil Canada, Vol.36, PP.171-83,2014.

[3] D.P.GUPTA, H.P.GUPTA, ZAIDI, D.K.SAXENA. Accuracy in estimation of gestational age in third trimester by fetal kidney length in Indian women, Indian. J Clin Pract, Vol. 2, PP.495-463,2013.

[4] N.SHIVALINHAIAH, K.SOWMYA, K.ANANYA. Fetal kidney length as a parameter of determination of gestational age in pregnancy. Int J Reprod Contracep Obstet Gynecol, Vol. 3, PP.424-427 .2014.

[5] M.E.ABDELRAZEK ABO-DONIA, I.E.SHALABY. Determination of gestational age by ultrasonographic measurement of fetal kidney length during third trimester of pregnancy. J Med Sci Res, Vol.2, PP.148$51,2019$.

[6] K.KUMAR, R.LALWANI, R.BABU. Ultrasonographic estimation of fetal gestational age by fetal kidney length. J Anat Soc India; 62:33-36,2013.

[7] M.PETER, A.K.NAYAK, P.P.GIRI. Fetal age length as a parameter for determination of gestational age from 20th to term in healthy women with uncomplicated pregnancy. Int $\mathbf{J}$ Res Med Sci, Vol.5, PP.1869-1873,2017.

[8] L.BERTAGNOLI, F.LALATTA, M.D.GALLICCHIO. Qualitative characterization of the growth of fetal kidney. J. Clin. Ultrasound. Vol.11(7), PP.349$56,1983$.

[9] J.P.EDEVBIE, A.O.AND. AKHIGBE. Ultrasound measurement of fetal kidney length in normal pregnancy and correlation with gestational age. Niger J Clin Pract, Vol. 21, PP.960-6,2018.

[10]A.GARG, N.GOYAL, R.K.GOREA, N.PATHAK. Normogram from fetal kidney length bu ultrasonographically. Journal of Punjab Academy of Forensic Medicine \& Toxicology, Vol.15, PP.14-16,2015.

[11] J.J.KANSARIA, S.V.PARULEKAR. Nomogram for fetal kidney length. Bombay Hosp J, Vol.51(2), PP.155-62,2009.

[12] M.G.UGER, A. MUSTAFA, H.C. OZCAN. Fetal kidney length as a useful adjacent parameter for better determination of gestational age. Saudi Med J, Vol. 5, PP.533$537,2016$.

[13] I.KAUL, V.MENIA, A.K.ANAND. Role of fetal kidney length in estimation of gestational age. JK Science. Apr-Jun, Vol.5, PP.65$69,2012$.

[14] J.C.KONJE， K.R.ABRAMS， S.C.BELL. Determination of gestational age after 24th weeks of gestation from fetal kidney measurement. Ultrasound Obstet Gynecol, Vol.19, PP.592-597,2002. 